\title{
Aesthetic Differences and Cultural Interpretation of Chinese and Western Music Forms
}

\author{
Guozhi Chen \\ Sichuan University of Arts and Science \\ Dazhou, China 635000
}

\begin{abstract}
Due to the differences in the geographical environment and cultural tradition, the Chinese and Western peoples have different musical aesthetic psychology characteristics. On the appearance of the external form, there are sharp differences in the aspects of texture, mode, rhythm, structure, etc. between the Chinese and Western traditional music. This difference is not the difference between superiority and inferiority, or the difference between advancedness and backwardness. They are all "flowers of civilization" born under their respective cultural backgrounds and have the same artistic value.
\end{abstract}

Keywords-music form; aesthetic differences; cultural interpretation

\section{INTRODUCTION}

Music is the art of sound. However, the sound is not only accepted by the human auditory sensor as the physical movement of the sound waves, but also as a form of externalization of musical psychological emotional image. [1] Due to the differences in the natural environment and cultural traditions, the Chinese and Western people have different "musical psychological emotional images". On the appearance of "extrinsic formation", it shows the significant differences in the weave, style, rhythm, structure and other forms between traditional Chinese and Western traditional music. The reasons for this difference can only be explained from the perspective of the culture.

\section{The AESTHETIC DifFERENCES AND CUlTURAL INTERPRETATION OF THE FABRIC}

The so-called "fabric" is the performance of the composition of the voice part. In general, Chinese music is dominated by monophonic fabric, which reflects unidirectional horizontal linearity in the thinking of the fabric. Although there is occasional "harmony", it is only the combination of natural sounds. There is no such concept of "harmony" as Western music emphasizes longitudinal combination and horizontal connection, and it also has no tendency. And it has no theoretical system to study the "harmony". Generally speaking, this "harmony" of Chinese music is still "monophone structure". The western music is dominated by multi-voice part. In the thinking of the fabric, it shows criss-cross mesh and three-dimensional shape. As early as the Renaissance, the western gradually formed the theoretical rules of the multi-voice combination and emerged the advanced polyphony genre of "Fugue". In the seventeenth and eighteenth centuries, "harmony acoustics" appeared which specialized in the longitudinal and transverse connection between sound and voice.

The differences between Chinese and Western music have long drawn people's attention and made an explanation. The famous Chinese musicologist Shen Zhibai once wrote that in the history of western music development, the chorus of men and women has a long tradition. Especially in the Christian church in Middle Ages, all the men and women should sing the hymn together, which created a good condition for the multi-part music. Due to physical differences, it is difficult for men and women to sing together in parallel or octave parallel chorus. In order to overcome this difficulty, they gradually adopted the two-part singing method of parallel fourth and fifth between men and women. [2] This can be said that the sprouting of western multi-part music, which laid the foundation for the extensive use of harmony in the later period.

On the contrary, in China, due to the long-term shackles of feudal ethics and the self-sufficient small-peasant economy for thousands of years, men and women singing and dancing together could rarely be seen since the Han dynasty. And it is difficult to produce multi-part music. In the meantime, Chinese opera sings with false voice, which blurs the boundaries of men and women. There is no difference between the tone and the voice zone. It is very difficult to produce the harmony similar to the Western. In addition, the Chinese are deeply influenced by Confucianism and Taoism. And they advocate the philosophy of "conforming to nature, unity of heaven and man". They pursue the philosophy of doctrine of the mean and peaceful environment. And the pentatonic single melody lines are suitable for the pursuit and mood. Therefore, China has the sprout in harmony at least during the Pre-qin period and even during the Shang and Zhou dynasties. [3] However, it did not give birth to harmony art in the West.

\section{THE AESTHETIC DIFFERENCES AND CUlTURAL INTERPRETATION OF THE MODE}

The core of the Chinese music mode system is the triad, which is the tonal structure combination of three tones according to certain interval relationship. These three tones can be assembled into different types. They can form five different types of pentatonic modes with distinctive Chinese 
characteristics: Do Re Mi Sol La. In addition to pentatonic mode, China also has heptachord. However, it is quite different from the Western heptachord. China's five tons of Do Re Mi Sol La can be regarded as "positive tone", and the other tones can be called "partial tones". The partial tone is usually just used as the crosstalk and the auxiliary tone among the positive tones, etc. It is a kind of decoration of the positive sound. And it still takes the pentatonic mode as the backbone, three panels as the core to expand the melody.

The origin and core of western musical mode system is four tonreihes formed by the connection of musical interval in ancient Greece. Each four tonreihes contain two whole tones and one semitone. And they are arranged in groups according to different intervals. Then, it forms several different types of four tonreihes. In the middle age, these different four tonreihes evolved into the formation of 12 kinds of modes, including 6 positive modes and 6 vicemodes. And these modes were later known as the "medieval mode" or "church mode". After the Renaissance, twelve kinds of modes evolved into major cycle mode and minor mode. These modes are based on heptachord.

There is no minor second and tritone, and the pentatonic scale will not produce the increasing or decreasing interval. And it would have no the sense of disharmony. The tendencies, functions and orientations of the voice are not strong. The voice is simpler and peaceful. Due to increasing or decreasing interval, the heptachord system has the sense of disharmony. With the semitones, the tendencies, functions and orientations of the voice are strong. Then, music is more complex, rich and refined.

In addition, most of the traditional Chinese music is presented in the same mode. Even if there is a change of mode, the majority of Chinese traditional music is mainly transferred within the same system. However, the changes of heteroplastic system are rare. The Western music always regards the tonality as the expansion of chords. The melody has become horizontal derivative of harmony. The harmony rules have become the order of melody. Therefore, the melody created by western music major, even art songs, popular songs and children's songs often have the change of tonality. The modulation has become a very important tool for the development of western music. The conservative development of Chinese music makes the music more "natural". With the tuning changes of same system, it would use the same sound level. If it only changes the order and primary and secondary of the sound, it would be more "intimate". However, the modulation of western music and the change of "tonality" are the changes of modes at different heights. And the sound would have the corresponding changes. It would be more estranged and unnatural.

There is no doubt that the differences in mode between Chinese and Western music originated from their different cultural backgrounds and aesthetic pursuits. Chinese culture is affected by "Golden Mean" of Confucianism and "quiet and inaction" thoughts of Taoist. The pentatomic scales are more harmonious and more in line with the aesthetic needs of literati and physicians. They pursue the natural, tranquil and quiet environment. In the Chinese music activities, there have been heptachord and twelve-tone columns. However, Chinese music did not choose them. And Chinese music chose the pentatonic system. [4] This is undoubtedly influenced by the Chinese traditional culture. Westerners tend to be exposed, radical, publicity, adventurous, rebellious and competitive. Therefore, they pursue the diversification of music emotions and the strong contrast and conflicts of music. The choice of heptachord and the normal use of tuning mode adapted to their aesthetic needs.

\section{AESTHETIC DIFFERENCES OF RHYTHM BETWEEN CHINESE AND WESTERN MUSIC}

There is also an important difference in rhythm between Chinese and Western music system. Western music is affected by the theory of "all things are number" of the ancient Greek Pythagorean and Christian chorus practical needs. In the 11th, 12th century, it developed a rhythm pattern, and invented mensural notation in the thirteenth century. It gradually divided the rhythm unit of music into precise time concepts, that is, whole notes, minim, quarter note, eighth note, and sixteenth notes, etc. And the segmentation of time length between the beat and the sound is very accurate. The intensity of its beat alternately appears regular and uniform. The rhythm is strong and distinct. This rigid beat has become a typical feature of Western music rhythms, known as the "functional rhythmicity movements". [5]

In traditional Chinese music, the functional rhythmicity movements are rare. At the beginning and at the end of much traditional Chinese music, we can often see the "saban" rhythms. That is, without bar lines and regular rhythms, the performer would flexibly adjust the rhythms according to the performance needs. Although some works have the bar lines. Its alternation of strength and weakness is not fixed as the Western music. The strong beat location is volatile and varied. In the Guqin music, which is the representative of traditional Chinese music, Guqin Jianzi notation is not used to mark or explain the rhythm at all. Instead, the performer "plays the game" and set the rhythm by themselves. This flexible and scalable rhythm is a typical feature of Chinese rhythms.

The differences in rhythmic features exhibited by Chinese and Western music are still caused by different cultural backgrounds and the different aesthetic psychology. Music is closely related to the languages of poetry in literature. Western languages focus on analysis and synthesis. They have strong sense of rhythm. And their poetries focus on the modulation. Westerners have always had the logic and rules-oriented tradition. Therefore, their music gradually abstracts various rhythmic patterns from the languages. And eventually, it forms the "rigid" rhythm of western music. Chinese traditional music is also influenced by the Chinese language. In Chinese ancient times, the monosyllable syllables often express different meanings. The poetry focus on the flat tone and the rhythm of the language is weak. The language characteristic of Chinese words is to emphasize words or sentences, which should be lengthened, aggravated and elevated. On the contrary, they should be lowered, relaxed and shortened. Therefore, there are the length and 
accent in Chinese traditional music. There is no clear measure and accent in the traditional music. And it is the same as the characteristics of the Chinese language. The thinking activities of the oriental ethnic groups are not abstract. In traditional Chinese music, although there are various decorative rhythms, and they have not formed the typical models. In the long run, Chinese music has formed a "rubbery" and "flexible" rhythm.

\section{THE AESTHETIC DifFERENCES BETWEEN CHINESE AND WESTERN MUSIC STRUCTURE}

Due to different cultural backgrounds, Chinese and Western music also show essential differences in structural thinking and aesthetic psychology. Since the Qin dynasty, the Chinese have been in a long-term feudal society, and their ideology of "unification" has been deeply rooted. The traditional way of thinking also emphasizes on the overall feeling of intuition. It treats individuals, nature and society as a whole. And it pays attention to universal connection in all aspects of things. Although the Chinese people do not reject the opposition, they place greater emphasis on the aspect of "unification." At the same time, the Chinese nation is also deeply influenced by the aesthetic view of "harmony," pursuing the golden mean of neutrality, the beauty of neutralization, and "keeping faith in ancient things." They are not good at innovation and difference. Therefore, Chinese people accept things with gradual mentality. In the aesthetic mentality of national music, the principle of "gradual" structure development of Chinese music has been formed. That is the structural thinking of music in more intuitive. The internal music lacks the logics and rational structure. The contrast and sub-paragraph is not obvious. Most traditional Chinese music is based on a fluid and gradual process of natural evolution. The theme of music does not emphasize opposites, conflicts, or drastic dramas, but pursues non-oppositional harmony. [6]

Due to environmental and historical impact, Westerners are outgoing and full of passion. They advocate treason, newness and competition. They have strong offensive. Reflected in the aesthetic psychology of national music, it forms the "mutation" structure development principles of the Western music. And it pursues unity in contrast. And it emphasizes the contrast. This influences of aesthetic awareness on the thinking of Western musical structure prominently are reflected in many types of music structure, such as single two, single three, complex trilogy and sonata, rhapsody and variations style. It reflects three principles of "expression - contrast - reproduction". However, the multisegment or juxtaposed music without reproduction has smaller application range to the music with reproduction. In fact, the difference of "gradual change" and "sudden change" is reflected in many other forms of Chinese and Western music, such as the development of melody, the intensity and speed of melody. And cultural background is closely related to the differences.

\section{CONCLUSION}

Through the above four aspects of the comparison and cultural interpretation of the aesthetic differences between Chinese and Western traditional music forms, it not only helps us to understand the essential forms of the music accurately and clearly, but also makes us see the distinctions of the forms and aesthetic characteristics between the traditional Chinese music and Western national music. However, this difference is not disparity. The contemporary French famous anthropologist Levi Strauss once said: "There is neither superiority nor inferiority of mankind, and no distinction among the cultures. Human cultures could be divided into many kinds, but be equal in value." In protecting traditional musical cultural characteristics, we must base on the aesthetic charm of Chinese nation and uphold its unique and strong aesthetic taste. At the same time, we must also actively learn from and absorb the achievements of other ethnic groups. It would integrate the nationality with the world. And then, the healthy and development of Chinese traditional music can be achieved.

\section{REFERENCES}

[1] Luo Xiaoping. The Aesthetics of Music [M]. Shanghai: Shanghai Music Publishing House, 2002: 272.

[2] Shen Zhibai. The reasons for the underdevelopment of the harmony in China in the past [J]. Musical Arts, 1982 (1): 16-21.

[3] Sun Weiquan. The origin of the concept of harmony in China [J] Musical Arts, 1986 (2): p.22-23, p.14.

[4] Liu Chenghua. Humanistic Interpretation of Chinese Music [M] Shanghai: Shanghai Music Publishing House, 2002: 71.

[5] Liu Chenghua. Humanistic Interpretation of Chinese Music [M] Shanghai: Shanghai Music Publishing House, 2002: 72.

[6] Shi Yong. An Introduction to Chinese Aesthetic Psychology of Music [M]. Shanghai: Shanghai Music Publishing House, 2008: 137. 